\title{
Theory Analysis of Left-Handed Grünwald-Letnikov Formula with $0<\alpha<1$ to Detect and Locate Singularities
}

\author{
Shaoxiang $\mathrm{Hu}^{1}$ and Ping Liang ${ }^{2}$ \\ ${ }^{1}$ School of Automation Engineering, University of Electronic Science and Technology of China, Chengdu, Sichuan 611731, China \\ ${ }^{2}$ Ningbo Xingaoyi Co., Ltd., No. 555 Yeshan Road, Yuyao City, Zhejiang 315400, China \\ Correspondence should be addressed to Ping Liang; clarke.liang@gmail.com
}

Received 21 January 2014; Accepted 9 March 2014; Published 3 April 2014

Academic Editor: Zhiwu Liao

Copyright (c) 2014 S. Hu and P. Liang. This is an open access article distributed under the Creative Commons Attribution License, which permits unrestricted use, distribution, and reproduction in any medium, provided the original work is properly cited.

\begin{abstract}
We study fractional-order derivatives of left-handed Grünwald-Letnikov formula with $0<\alpha<1$ to detect and locate singularities in theory. The widely used four types of ideal singularities are analyzed by deducing their fractional derivative formula. The local extrema of fractional derivatives are used to locate the singularities. Theory analysis indicates that fractional-order derivatives of left-handed Grünwald-Letnikov formula with $0<\alpha<1$ can detect and locate four types of ideal singularities correctly, which shows better performance than classical 1-order derivatives in theory.
\end{abstract}

\section{Introduction}

How to preserve singularities in image and signal processing is a very important problem [1-5]. Recently, fractional derivatives become very important tools in the field [6-17]. All of the methods based on fractional derivatives reported good processing results by modifying some classical partial differential equations (PDEs) to fully or partial fractionalorder derivative PDEs. Just as the recent theory analysis efforts in fractional derivatives by mathematicians [18-20], all theory analyses focus on how to approach the PDEs and how to find their exact solutions.

However, we think fractional derivatives should be studied differently. It is well known that one powerful method to preserve singularities in signal processing is to detect and locate singularities correctly and then to protect them in signal processing. Thus, we think the most important problem in theory analysis should be if the fractional derivatives can detect and locate singularities well.

In this paper, we study fractional-order derivatives of left-handed Grünwald-Letnikov formula with $0<\alpha<1$ to detect and locate the widely used four types of ideal singularities in theory. Theory analysis is from deducing the fractional derivatives of four types of ideal singularities with an indicated singularity in each case. The differences of these fractional derivative values are studied to find the local extrema. The extrema are considered as the singularities.

The rest of this paper is as follows. Section 2 introduces some basic theory backgrounds in fractional derivatives; we also deduce some useful results based on these theory backgrounds. In Section 3, we introduce the 1-order differential method used in singularity detection and location, and the detected and located results of four types of ideal singularities are also presented. Section 4 discusses the steps in singularities detection and location using fractional derivatives, and then they are used to detect and locate the four types of ideal singularities. We also give conclusions and acknowledgments finally.

\section{Fractional Derivatives}

In contrast to integer-order differentials $d^{n} / d t^{n}$, fractionalorder derivatives are defined as operators whose orders have been extended to noninteger numbers. There are a number of definitions of fractional derivatives. The usual way of representing the fractional derivatives is by the left-handed Riemann-Liouville formula (R-L formula). 
The left-handed R-L formula of order $\alpha$, for $x \in[a, b]$, is defined by $[21,22]$

$$
D_{\mathrm{RL}}^{\alpha} u(x)=\frac{1}{\Gamma(n-\alpha)} \frac{d^{n}}{d x^{n}} \int_{a}^{x} u(\tau)(x-\tau)^{n-\alpha-1} d \tau,
$$

where $n-1<\alpha<n$ and $n=[\alpha]+1$, with $[\alpha]$ denoting the integer part of $\alpha$, and $\Gamma(\cdot)$ is the Gamma function defined as

$$
\Gamma(z)=\int_{0}^{\infty} t^{z-1} e^{-t} d t
$$

Another way to represent the fractional derivatives is by the Grünwald-Letnikov (G-L) formula, which is a generalization of the ordinary discretization formulas for integer-order derivatives. For $\alpha>0$, the left-handed G-L formula is

$$
D_{\mathrm{GL}}^{\alpha} u(x)=\lim _{\Delta x \rightarrow 0} \frac{1}{\Delta x^{\alpha}} \sum_{k=0}^{[(x-a) / \Delta x]} \omega_{k}^{(\alpha)} u(x-k \Delta x)
$$

where $\Delta x$ denotes the uniform space step and $\omega_{k}^{(\alpha)}=$ $(-1)^{k}\left(\begin{array}{l}\alpha \\ k\end{array}\right)$ represents the normalized G-L weights that are given by

$$
\begin{gathered}
\omega_{0}^{(\alpha)}=1, \\
\omega_{k}^{(\alpha)}=(-1)^{k} \frac{\alpha(\alpha-1) \cdots(\alpha-k+1)}{k !} \\
=\frac{\Gamma(k-\alpha)}{\Gamma(-\alpha) \Gamma(k+1)}, \quad \text { for } k=1,2,3, \ldots
\end{gathered}
$$

The above two definitions have different forms. However, by requiring a reasonable behavior of the function $u(x)$ and their derivatives, we can relate the two definitions with the following proposition $[21,23]$.

Proposition 1. Let us assume that the function $u(x)$ is $(n-1)$ times differential in $[a, b]$ and that the nth derivative of $u(x)$ is integrable in $[a, b]$. Then, for every $n-1<\alpha<n$, one has

$$
D_{G L}^{\alpha} u(x)=D_{R L}^{\alpha} u(x), \quad a \leq x \leq b .
$$

Generally, the analytic definition given by (1) is used in the formulation of the fractional partial differential equations (PDEs), while G-L definitions in (3) are maybe used to discretize the fractional PDEs to obtain a numerical solution. Since the problem in this paper has discrete form, G-L formula defined in (3) will be adopted in our following discussion.

Remark 2. For $\alpha=1,(1)$ and (3) become the classical first derivative, and, for any $\alpha=n, n \in \mathcal{\aleph}$ is a positive integer; they are classical $n$th derivatives of $u(x)$ if the function $u(x)$ is $(n-1)$ times differentiable. Note that, for $\alpha$ is a positive integer, the equations are with limit support whose support length is $\alpha+1$. However, for $\alpha$ is not an integer, fractional derivatives defined above are nonlocal operators. That is, the value of the fractional derivative at a point $x$ depends on the function values at all the points to the left of the point $x$.
Therefore, in order to handle fractional derivative numerically, it is necessary to compute the coefficients $\omega_{k}^{(\alpha)}$, where $\alpha$ is the order of the fractional derivative. For that, we can use the recurrence relationships presented in Lemma 3.

Lemma 3. The recurrence relationship of the coefficients of $G$ $L$ formula $\omega_{k}^{(\alpha)}$ defined in (3) is

$$
\omega_{0}(\alpha)=1 ; \quad \omega_{k}^{(\alpha)}=\left(1-\frac{\alpha+1}{k}\right) \omega_{k-1}^{(\alpha)}, \quad k=1,2,3, \ldots
$$

Proof. For $k=0, \omega_{0}(\alpha)=1$.

Assume that when $n=k-1$, we have

$$
\omega_{k-1}^{(\alpha)}=\left(1-\frac{\alpha+1}{k-1}\right) \omega_{k-2}^{(\alpha)}
$$

For $n=k$,

$$
\begin{aligned}
\omega_{k}^{(\alpha)} & =\frac{\Gamma(k-\alpha)}{\Gamma(-\alpha) \Gamma(k+1)}=\frac{(k-1-\alpha) \Gamma(k-1-\alpha)}{k \Gamma(-\alpha) \Gamma(k)} \\
& =\left(1-\frac{\alpha+1}{k}\right) \omega_{k-1}^{(\alpha)} .
\end{aligned}
$$

Therefore, for all $k=1,2,3, \ldots$, we have

$$
\omega_{k}^{(\alpha)}=\left(1-\frac{\alpha+1}{k}\right) \omega_{k-1}^{(\alpha)} .
$$

Lemma 4. The nonlocal operator defined in (3) is a linear operator.

Proof. $u(x)$ and $v(x)$ are two functions, and $x \in[a, b], \lambda$, is a real number, since

(1)

$$
\begin{aligned}
D_{\mathrm{GL}}^{\alpha}[u(x)+v(x)]= & \lim _{\Delta x \rightarrow 0} \frac{1}{\Delta x^{\alpha}} \sum_{k=0}^{[(x-a) / \Delta x]} \omega_{k}^{(\alpha)} \\
& \times[u(x-k \Delta x)+v(x-k \Delta x)] \\
= & \lim _{\Delta x \rightarrow 0} \frac{1}{\Delta x^{\alpha}} \sum_{k=0}^{[(x-a) / \Delta x]} \omega_{k}^{(\alpha)} u(x-k \Delta x) \\
& +\lim _{\Delta x \rightarrow 0} \frac{1}{\Delta x^{\alpha}} \sum_{k=0}^{[(x-a) / \Delta x]} \omega_{k}^{(\alpha)} v(x-k \Delta x) \\
= & D_{\mathrm{GL}}^{\alpha} u(x)+D_{\mathrm{GL}}^{\alpha} v(x),
\end{aligned}
$$

(2)

$$
\begin{aligned}
D_{\mathrm{GL}}^{\alpha}[\lambda u(x)] & =\lim _{\Delta x \rightarrow 0} \frac{1}{\Delta x^{\alpha}} \sum_{k=0}^{[(x-a) / \Delta x]} \omega_{k}^{(\alpha)}[\lambda u(x)] \\
& =\lambda \lim _{\Delta x \rightarrow 0} \frac{1}{\Delta x^{\alpha}} \sum_{k=0}^{[(x-a) / \Delta x]} \omega_{k}^{(\alpha)} u(x) \\
& =\lambda D_{\mathrm{GL}}^{\alpha} u(x) .
\end{aligned}
$$


Lemma 5. Consider $\sum_{k=0}^{\infty} \omega_{k}^{(\alpha)}=0$.

Proof. Since $(1-z)^{\alpha}=\sum_{k=0}^{\infty} \omega_{k}^{(\alpha)} z^{k}$, let $k=1$, and we have

$$
\sum_{k=0}^{\infty} \omega_{k}^{(\alpha)}=(1-1)^{\alpha}=0
$$

Lemma 6. For $0<\alpha<1$, $t$ is a positive integer, $m \geq t$, and one has

$$
\omega_{k}^{(\alpha)}<0, \quad k=1,2,3, \ldots,
$$

and $\sum_{k=t}^{\infty} \omega_{k}^{(\alpha)}<0, \sum_{k=t}^{m} \omega_{k}^{(\alpha)}<0$.

Proof. For $k=1, \omega_{1}^{(\alpha)}=-\alpha<0$.

Assume that $\omega_{k}^{(\alpha)}<0$.

According to Lemma 3, we have $\omega_{k+1}^{(\alpha)}=(1-((\alpha+1) /(k+$ $1))) \omega_{k}^{(\alpha)}$. Since $0<\alpha<1$ and $k+1 \geq 2,(1-((\alpha+1) /(k+1)))>$ 0 . Thus, $\operatorname{sgn}\left(\omega_{k+1}^{(\alpha)}\right)=\operatorname{sgn}\left(\omega_{k}^{(\alpha)}\right)<0$. Here,

$$
\operatorname{sgn}(x)= \begin{cases}1, & x \geq 0 \\ -1, & x<0\end{cases}
$$

Then we have $\omega_{k+1}^{(\alpha)}<0$. Thus $\sum_{k=t}^{\infty} \omega_{k}^{(\alpha)}<0, \sum_{k=t}^{m} \omega_{k}^{(\alpha)}<0$.

Lemma 7. For $0<\alpha<1, t \geq 0$ is an integer,

$$
\sum_{k=0}^{t} \omega_{k}^{(\alpha)}>0
$$

Proof. According to Lemma 5, we have

$$
0=\sum_{k=0}^{\infty} \omega_{k}^{(\alpha)}=\sum_{k=0}^{t} \omega_{k}^{(\alpha)}+\sum_{k=t+1}^{\infty} \omega_{k}^{(\alpha)} .
$$

According to Lemma $6, \sum_{k=t+1}^{\infty} \omega_{k}^{(\alpha)}<0$. Therefore, $\sum_{k=0}^{t} \omega_{k}^{(\alpha)}>0$.

Lemma 8. For $0<\alpha<1, m \geq 0$ is an integer and t is a positive integer

$$
\begin{gathered}
\sum_{k=m}^{t} \omega_{k}^{(\alpha)}(-k)>0, \\
\sum_{k=m}^{\infty} \omega_{k}^{(\alpha)}(-k)>0 .
\end{gathered}
$$

Proof. From Lemma 6, for $0<\alpha<1, \omega_{k}^{(\alpha)}<0, k=1,2,3, \ldots$.

Thus, $\omega_{k}^{(\alpha)}(-k)>0$ for $k=1,2,3, \ldots$, and $t>0$. We have $\sum_{k=m}^{\infty} \omega_{k}^{(\alpha)}(-k)>0, m \geq 1$, and $\sum_{k=m}^{t} \omega_{k}^{(\alpha)}(-k)>0, m \geq 1$, $t \geq 1$.

\section{Classical Singularity Detection and Location}

Singularity detection is the name for a set of mathematical methods which aim at identifying points in a digital signal at which the signal value changes sharply or, more formally, has discontinuities.

3.1. Singularity Types. We can categorize singularities as step, roof, jump, and ramp. They can be represented as

(1) ideal step

$$
s(x)= \begin{cases}1, & x \geq x_{0}, \\ 0, & x<x_{0},\end{cases}
$$

(2) ideal roof

$$
s(x)= \begin{cases}m\left(x-x_{0}\right)+c_{0}, & x_{0}-h_{1}<x \leq x_{0}, \\ n\left(x-x_{0}\right)+c_{0}, & x_{0}<x<x_{0}+h_{2},\end{cases}
$$

(3) ideal impulse

$$
s(x)= \begin{cases}b, & x=x_{0}, \\ 0, & x \neq x_{0},\end{cases}
$$

(4) ideal ramp

$$
s(x)= \begin{cases}m\left(x-x_{0}\right)+c_{0}, & x_{0}-h_{1}<x \leq x_{0}, \\ c_{0}, & x>x_{0} .\end{cases}
$$

\section{Singularity Detection by 1-Order Derivatives}

Singularity detection is the name for a set of mathematical methods which aim at identifying points in a digital signal at which the signal value changes sharply or, more formally, has discontinuities.

4.1. The First-Order Derivatives for Singularities. The singularity detection by 1-order derivatives detects singularities by first computing a measure of singularity strength, usually a first-order derivative expression, and then searching for local absolute maxima as the locations of singularities. The simplest approach to compute first-order derivatives is to use left-handed differences

$$
D u(x)=u(x)-u(x-1) .
$$

Therefore, first-order derivatives of the four types of singularities are

(1) ideal step

$$
D s(x)= \begin{cases}0, & x<x_{0} \\ 1, & x=x_{0} \\ 0, & x>x_{0}\end{cases}
$$

(2) ideal roof

$$
D s(x)= \begin{cases}m, & x_{0}-h_{1}<x \leq x_{0}, \\ n, & x_{0}<x<x_{0}+h_{2},\end{cases}
$$


(3) ideal impulse

$$
D s(x)= \begin{cases}b, & x=x_{0} \\ -b, & x=x_{0}+1 \\ 0, & \text { otherwise }\end{cases}
$$

(4) ideal ramp

$$
D s(x)= \begin{cases}m, & x_{0}-h_{1}<x \leq x_{0} \\ 0, & x>x_{0} .\end{cases}
$$

Then, find absolute maxima of $D s(x)$ to locate the positions of singularities. Here, absolute maxima are the maxima of absolute values of $D s(x)$.

4.2. Detect and Locate Singularities. The main steps to locate singularities are as follows: (1) find all points with $D(s(x)) \neq 0$; (2) compute absolute values of $D(s(x))$; (3) if $D s(x)>0$, compare absolute values of $D s([x])$ and $D s([x+1])$, and if $a b s(D s(x))>a b s(D s(x+1)), x$ is the singularity; if $D s(x)<0$, compare absolute values of $D s([x])$ and $D s([x-1])$, and if $a b s(D s(x))>a b s(D s(x-1)), x$ is the singularity. Four types of ideal singularities are discussed as follows. Each one has a corresponding singularity on $x_{0}$.

Ideal Step. The absolute values of $D(s(x))$ are the same as $D(s(x))$. Therefore, the absolute maxima are 1 on the position $x_{0}$, and then compare absolute values of $D s\left(x_{0}\right)$ and $D s\left(\left[x_{0}+\right.\right.$ $1])$, and we have $D s\left(x_{0}\right)=1>\operatorname{Ds}\left(\left[x_{0}+1\right]\right)=0$. Therefore, the singularity is detected by 1-order derivative and is located on $x_{0}$, which means 1-order derivatives can detect and locate ideal step singularities correctly.

Ideal Roof. The absolute value of $D(s)$ is

$$
a b s(D s(x))= \begin{cases}a b s(m), & x_{0}-h_{1}<x \leq x_{0}, \\ a b s(n), & x_{0}<x<x_{0}+h_{2} .\end{cases}
$$

Here, $\operatorname{abs}(\cdot)$ is

$$
\operatorname{abs}(x)= \begin{cases}x, & x \geq 0 \\ -x, & x<0\end{cases}
$$

For $D(s(x))=m>0, x_{0}-h_{1}<x<x_{0}$, we should compare the absolute values of $D(s(x))$ and $D(s(x+1))$. Since $\operatorname{abs}(D(s(x)))=\operatorname{abs}(D(s(x+1)))=m$, all $x \in\left(x_{0}-h_{1}, x_{0}\right)$ are not singularities.

For $D(s(x))=m<0, x_{0}-h_{1}<x \leq x_{0}$, we should compare the absolute values of $D(s(x))$ and $D(s(x-1))$. Since $\operatorname{abs}(D(s(x)))=\operatorname{abs}(D(s(x-1)))=m$, all $x \in\left(x_{0}-h_{1}, x_{0}\right]$ are not singularities.

$D\left(s\left(x_{0}\right)\right)=m>0$, and we should compare the absolute values of $D\left(s\left(x_{0}\right)\right)$ and $D\left(s\left(x_{0}+1\right)\right)$. Since $a b s\left(D\left(s\left(x_{0}\right)\right)\right)=$ $a b s(m)$ and $a b s\left(D\left(s\left(x_{0}+1\right)\right)\right)=a b s(n)$, if $a b s(m)>a b s(n)$, $x_{0}$ is the singularity; otherwise, $x_{0}$ is not a singularity.

$D\left(s\left(x_{0}+1\right)\right)=n<0$, and we should compare the absolute values of $D\left(s\left(x_{0}+1\right)\right)$ and $D\left(s\left(x_{0}\right)\right)$. Since $a b s\left(D\left(s\left(x_{0}\right)\right)\right)=a b s(m)$ and $a b s\left(D\left(s\left(x_{0}+1\right)\right)\right)=a b s(n)$, if $\operatorname{abs}(m)<a b s(n), x_{0}+1$ is the singularity; otherwise, $x_{0}+1$ is not a singularity.

For $D(s(x))=n<0, x_{0}+1<x<x_{0}+h_{2}$, we should compare the absolute values of $D(s(x))$ and $D(s(x-1))$. Since $\operatorname{abs}(D(s(x-1)))=a b s(D(s(x)))=a b s(n), x \in\left(x_{0}+1, x_{0}+h_{2}\right)$ are not the singularities.

For $D(s(x))=n>0, x_{0}+1 \leq x<x_{0}+h_{2}$, we should compare the absolute values of $D(s(x))$ and $D(s(x+1))$. Since $\operatorname{abs}(D(s(x+1)))=a b s(D(s(x)))=a b s(n), x \in\left[x_{0}+1, x_{0}+h_{2}\right)$ are not the singularities.

Ideal Impulse. $(D(s))$ has two nonzeros, that is, $x=x_{0}$ and $x=x_{0}+1$. For $D\left(s\left(x_{0}\right)\right)=b>0$, comparing the absolute values of $D\left(s\left(x_{0}\right)\right)$ and $D\left(s\left(x_{0}+1\right)\right)$, we have $a b s\left(D\left(s\left(x_{0}\right)\right)\right)=$ $\operatorname{abs}\left(D\left(s\left(x_{0}+1\right)\right)\right)=a b s(b)$. Thus, $x_{0}$ is not the singularity. For $D\left(s\left(x_{0}\right)\right)=b<0$, comparing the absolute values of $D\left(s\left(x_{0}\right)\right)$ and $D\left(s\left(x_{0}-1\right)\right)$, we have $a b s\left(D\left(s\left(x_{0}\right)\right)\right)=a b s(b)>$ $a b s\left(D\left(s\left(x_{0}-1\right)\right)\right)=0$. Thus, $x_{0}$ is the singularity.

For $D\left(s\left(x_{0}+1\right)\right)=-b<0$, comparing the absolute values of $D\left(s\left(x_{0}+1\right)\right)$ and $D\left(s\left(x_{0}\right)\right)$, we have $a b s\left(D\left(s\left(x_{0}+1\right)\right)\right)=$ $\operatorname{abs}\left(D\left(s\left(x_{0}\right)\right)\right)=a b s(b)$. Thus, $x_{0}+1$ is not the singularity. For $D\left(s\left(x_{0}+1\right)\right)=-b>0$, comparing the absolute values of $D\left(s\left(x_{0}+1\right)\right)$ and $D\left(s\left(x_{0}+2\right)\right)$, we have $a b s\left(D\left(s\left(x_{0}+1\right)\right)\right)=$ $a b s(b)>a b s\left(D\left(s\left(x_{0}+2\right)\right)\right)=0$. Thus, $x_{0}+1$ is the singularity.

In summary, for $b>0$, no singularity is detected; for $b<$ 0 , both $x_{0}$ and $x_{0}+1$ are located as singularities.

Ideal Ramp. The absolute value of $D(s)$ is

$$
\operatorname{abs}(D s(x))= \begin{cases}m, & x_{0}-h_{1}<x \leq x_{0} \\ 0, & x>x_{0} .\end{cases}
$$

For $D(s(x))=m>0, x_{0}-h_{1}<x<x_{0}$, we should compare the absolute values of $D(s(x))$ and $D(s(x+1))$. Since $\operatorname{abs}(D(s(x)))=a b s(D(s(x+1)))=a b s(m)$, all $x_{0}-h_{1}<x<$ $x_{0}$ are not singularities.

For $D(s(x))=m<0, x_{0}-h_{1}<x \leq x_{0}$, we should compare the absolute values of $D(s(x))$ and $D(s(x-1))$. Since $\operatorname{abs}(D(s(x)))=a b s(D(s(x+1)))=a b s(m)$, all $x_{0}-h_{1}<x \leq$ $x_{0}$ are not singularities.

$D\left(s\left(x_{0}\right)\right)=m>0$, and we should compare the absolute values of $D\left(s\left(x_{0}\right)\right)$ and $D\left(s\left(x_{0}+1\right)\right)$. Since $a b s\left(D\left(s\left(x_{0}\right)\right)\right)=m$ and $\operatorname{abs}\left(D\left(s\left(x_{0}+1\right)\right)\right)=0, x_{0}$ is the singularity. Thus, when $m>0$, ideal ramp singularity can be detected and located correctly by 1-order derivatives.

Summarizing the above conclusion, we have the following.

Theorem 9. The detection and location of four types of ideal singularities using 1-order derivatives are

(1) ideal step: 1-order derivative can detect and locate ideal step singularities correctly;

(2) ideal roof: 1-order derivative can detect ideal roof singularities when $a b s(m) \neq a b s(n)$. But, for $a b s(m)<$ abs $(n)$, a false singularity $x_{0}+1$ is located;

(3) ideal impulse: the singularity can not be detected; 
(4) ideal ramp: 1-order derivative can detect and locate ideal ramp singularities correctly when $m>0$, for $m<0$, and the singularity can not be detected.

\section{Singularity Detection by Fractional-Order Derivatives}

The singularity detection by fractional-order derivatives detects singularities by computing fractional-order derivative expression firstly and then searching for local extrema as the locations of singularities.

5.1. Fractional Derivatives of Four Types of Singularities. Fractional-order derivatives of the four types of singularities are as follows.

Ideal Step. The fractional-order derivatives of ideal step singularities are

$$
D_{\mathrm{GL}}^{\alpha} s(x)= \begin{cases}\sum_{0}^{\left[x-x_{0}\right]} \omega_{k}^{(\alpha)}, & x \geq x_{0} \\ 0, & x<x_{0} .\end{cases}
$$

Ideal Roof. The fractional-order derivatives of ideal roof singularities are

$$
\begin{aligned}
& D_{\mathrm{GL}}^{\alpha} s(x) \\
& \quad \begin{cases}m \sum_{k=0}^{\left[x-x_{0}+h_{1}\right]} \omega_{k}^{(\alpha)}\left(-k+\left[x-x_{0}\right]\right) & x_{0}-h_{1}<x \leq x_{0} \\
+c_{0} \sum_{k=0}^{\left[x-x_{0}+h_{1}\right]} \omega_{k}^{(\alpha)}, & \\
\sum_{k=0}^{\left[x-x_{0}\right]-1} \omega_{k}^{(\alpha)}\left(-k+\left[x-x_{0}\right]\right) & x_{0}<x<x_{0}+h_{2} . \\
+m \sum_{k=\left[x-x_{0}\right]}^{\left[x-x_{1}\right]} \omega_{k}^{(\alpha)}\left(-k+\left[x-x_{0}\right]\right) & \\
+c_{0} \sum_{k=0}^{\left[x-x_{0}+h_{1}\right]} \omega_{k}^{(\alpha)}, & \end{cases}
\end{aligned}
$$

Ideal Ramp. The fractional-order derivatives of ideal ramp singularities are

$$
\begin{aligned}
D_{\mathrm{GL}}^{\alpha} s(x) & \\
= & \begin{cases}m \sum_{k=0}^{\left[x-x_{0}+h_{1}\right]} \omega_{k}^{(\alpha)}\left(-k+\left[x-x_{0}\right]\right) & x_{0}-h_{1}<x \leq x_{0} \\
+c_{0} \sum_{k=0}^{\left[x-x_{0}+h_{1}\right]} \omega_{k}^{(\alpha)}, & \\
m \sum_{k=\left[x-x_{0}+1\right]}^{\left[x-x_{0}+h_{1}\right]} \omega_{k}^{(\alpha)}\left(-k+\left[x-x_{0}\right]\right) & \\
+c_{0} \sum_{k=0}^{\left[h_{1}\right]} \omega_{k}^{(\alpha)}, & \end{cases}
\end{aligned}
$$

5.2. Find Extremum. The main steps to find extrema of fractional-order derivatives $D_{\mathrm{GL}}^{\alpha} s(x)$ with $0<\alpha<1$ are as follows: for all $D_{\mathrm{GL}}^{\alpha} s(x) \neq 0$, compare values of $D_{\mathrm{GL}}^{\alpha} s(x-1)$, $D_{\mathrm{GL}}^{\alpha} s(x)$, and $D_{\mathrm{GL}}^{\alpha} s(x+1)$. If $D_{\mathrm{GL}}^{\alpha} s(x)-D_{\mathrm{GL}}^{\alpha} s(x-1)>$ $t_{0}$ and $D_{\mathrm{GL}}^{\alpha} s(x)-D_{\mathrm{GL}}^{\alpha} s(x+1)>t_{0}$ or $D_{\mathrm{GL}}^{\alpha} s(x-1)-$ $D_{\mathrm{GL}}^{\alpha} s(x)>t_{0}$ and $D_{\mathrm{GL}}^{\alpha} s(x+1)-D_{\mathrm{GL}}^{\alpha} s(x)>t_{0}$, where $t_{0}$ is predefined threshold, $x$ is the singularity. Four types of ideal singularities are discussed as follows. Each one has a corresponding singularity on $x_{0}$.

Based on the above discussion, we can detect and locate four types of singularities as follows.

Ideal Step. Since $D\left(s\left(x_{0}\right)\right)=\omega_{0}^{(\alpha)}=1, D\left(s\left(x_{0}-1\right)\right)=0$ and $D\left(s\left(x_{0}+1\right)\right)=1+\omega_{1}^{(\alpha)}$. According to Lemma 6, $\omega_{1}^{(\alpha)}<0$ for $0<\alpha<1$. Thus, $D\left(s\left(x_{0}\right)\right)=1>D\left(s\left(x_{0}+1\right)\right)$ and $D\left(s\left(x_{0}\right)\right)=$ $1>D\left(s\left(x_{0}-1\right)\right)=0$. So, $x_{0}$ is the singularity. When $x>x_{0}$, $D(s(x-1)) \geq D(s(x))$ and $D(s(x+1)) \leq D(s(x))$, so all $x>x_{0}$ are not singularities.

From the summary above, there is only one singularity on $x_{0}$.

Ideal Roof. Since

$$
\begin{aligned}
D_{\mathrm{GL}}^{(\alpha)} s\left(x_{0}\right) & =m \sum_{k=1}^{\left[h_{1}\right]} \omega_{k}^{(\alpha)}(-k)+c_{0} \sum_{k=0}^{\left[h_{1}\right]} \omega_{k}^{(\alpha)}, \\
D_{\mathrm{GL}}^{(\alpha)} s\left(x_{0}-1\right) & =m \sum_{k=0}^{\left[h_{1}-1\right]} \omega_{k}^{(\alpha)}(-k-1)+c_{0} \sum_{k=0}^{\left[h_{1}-1\right]} \omega_{k}^{(\alpha)}, \\
D_{\mathrm{GL}}^{(\alpha)} s\left(x_{0}+1\right) & =n+m \sum_{k=2}^{\left[h_{1}+1\right]} \omega_{k}^{(\alpha)}(-k+1)+c_{0} \sum_{k=0}^{\left[h_{1}+1\right]} \omega_{k}^{(\alpha)},
\end{aligned}
$$

thus,

$$
\begin{aligned}
D_{\mathrm{GL}}^{(\alpha)} s\left(x_{0}\right)-D_{\mathrm{GL}}^{(\alpha)} s\left(x_{0}-1\right)= & m \sum_{k=0}^{\left[h_{1}-1\right]} \omega_{k}^{(\alpha)}+m \omega_{\left[h_{1}\right]}^{(\alpha)}\left(-\left[h_{1}\right]\right) \\
& +c_{0} \omega_{\left[h_{1}\right]}^{(\alpha)},
\end{aligned}
$$




$$
\begin{aligned}
D_{\mathrm{GL}}^{(\alpha)} s\left(x_{0}\right)-D_{\mathrm{GL}}^{(\alpha)} s\left(x_{0}+1\right)= & -n-m \sum_{k=1}^{\left[h_{1}\right]} \omega_{k}^{(\alpha)} \\
& +m \omega_{\left[h_{1}+1\right]}^{(\alpha)}\left[h_{1}\right]-c_{0} \omega_{\left[h_{1}\right]+1}^{(\alpha)} .
\end{aligned}
$$

The ideal roof implies that when $m>0$, we have $c_{0}>0, n<0$, and when $m<0$, we have $c_{0}<0, n>0$.

According to Lemma $6, \sum_{k=0}^{\left[h_{1}-1\right]} \omega_{k}^{(\alpha)}>0$ and, according to Lemma 7, $\omega_{\left[h_{1}\right]}^{(\alpha)}\left(-\left[h_{1}\right]\right)>0$. Moreover, $-m h_{1}+c_{0}=0$, Thus, the right hand of (35) is $m \sum_{k=0}^{\left[h_{1}-1\right]} \omega_{k}^{(\alpha)}$ and it has the same sign as $m$. That is, when $m>0$, it is a positive number while when $m<0$, it is a negative number. The right hand of (36) can be written as $-n-m \sum_{k=1}^{\left[h_{1}\right]} \omega_{k}^{(\alpha)}$, which also has the same sign as $m$. Therefore, when $m>0, D_{\mathrm{GL}}^{(\alpha)} s\left(x_{0}\right)$ is a maximum while when $m<0, D_{\mathrm{GL}}^{(\alpha)} s\left(x_{0}\right)$ is a minimum. In summary, $x_{0}$ is a singularity.

Ideal Impulse. For $x=x_{0}, D_{\mathrm{GL}}^{(\alpha)} s\left(x_{0}\right)=b, D_{\mathrm{GL}}^{(\alpha)} s\left(x_{0}-1\right)=0$, and $D_{\mathrm{GL}}^{(\alpha)} s\left(x_{0}+1\right)=\omega_{1}^{(\alpha)} b$. According to Lemma 5, $\omega_{1}^{(\alpha)}<0$ and $a b s\left(\omega_{1}^{(\alpha)}\right)<1$ for $0<\alpha<1$. Thus, when $b>0$, we have $D_{\mathrm{GL}}^{(\alpha)} s\left(x_{0}\right)-D_{\mathrm{GL}}^{(\alpha)} s\left(x_{0}-1\right)=b>0$ and $D_{\mathrm{GL}}^{(\alpha)} s\left(x_{0}\right)-D_{\mathrm{GL}}^{(\alpha)} s\left(x_{0}+\right.$ $1)=b\left(1-\omega_{1}^{(\alpha)}\right)>0$, while when $b<0$, we have $D_{\mathrm{GL}}^{(\alpha)} s\left(x_{0}\right)-$ $D_{\mathrm{GL}}^{(\alpha)} s\left(x_{0}-1\right)=b<0$ and $D_{\mathrm{GL}}^{(\alpha)} s\left(x_{0}\right)-D_{\mathrm{GL}}^{(\alpha)} s\left(x_{0}+1\right)=b(1-$ $\left.\omega_{1}^{(\alpha)}\right)<0$. Therefore, $x_{0}$ is the singularity.

Ideal Ramp. Since

$$
\begin{gathered}
D_{\mathrm{GL}}^{(\alpha)} s\left(x_{0}\right)=m \sum_{k=1}^{\left[h_{1}\right]} \omega_{k}^{(\alpha)}(-k)+c_{0} \sum_{k=0}^{\left[h_{1}\right]} \omega_{k}^{(\alpha)}, \\
D_{\mathrm{GL}}^{(\alpha)} s\left(x_{0}-1\right)=m \sum_{k=0}^{\left[h_{1}-1\right]} \omega_{k}^{(\alpha)}(-k-1)+c_{0} \sum_{k=0}^{\left[h_{1}-1\right]} \omega_{k}^{(\alpha)}, \\
D_{\mathrm{GL}}^{(\alpha)} s\left(x_{0}+1\right)=m \sum_{k=1}^{\left[h_{1}+1\right]} \omega_{k}^{(\alpha)}(-k+1)+c_{0} \sum_{k=0}^{\left[h_{1}\right]} \omega_{k}^{(\alpha)},
\end{gathered}
$$

according to the above discussion of ideal roof, we know that $D_{\mathrm{GL}}^{(\alpha)} s\left(x_{0}\right)-D_{\mathrm{GL}}^{(\alpha)} s\left(x_{0}-1\right)$ has the same sign as $m$. Thus, we only discuss $D_{\mathrm{GL}}^{(\alpha)} s\left(x_{0}\right)-D_{\mathrm{GL}}^{(\alpha)} s\left(x_{0}+1\right)$ as follows:

$$
\begin{aligned}
D_{\mathrm{GL}}^{(\alpha)} s\left(x_{0}\right)-D_{\mathrm{GL}}^{(\alpha)} s\left(x_{0}+1\right) & =m \sum_{k=1}^{\left[h_{1}\right]}\left(-\omega_{k}^{(\alpha)}\right)+m \omega_{\left[h_{1}\right]+1}^{(\alpha)}\left[h_{1}\right] \\
& =m \sum_{k=1}^{\left[h_{1}\right]}\left(\omega_{\left[h_{1}+1\right]}^{(\alpha)}-\omega_{k}^{(\alpha)}\right) .
\end{aligned}
$$

According to Lemma 4, $\omega_{\left[h_{1}\right]+1}^{(\alpha)}<0$, and $a b s\left(\omega_{\left[h_{1}\right]+1}^{(\alpha)}\right)<$ $\operatorname{abs}\left(\omega_{k}^{(\alpha)}\right)$, we have $\sum_{k=1}^{\left[h_{1}\right]}\left(\omega_{\left[h_{1}+1\right]}^{(\alpha)}-\omega_{k}^{(\alpha)}\right)>0$. Therefore, $D_{\mathrm{GL}}^{(\alpha)} s\left(x_{0}\right)-D_{\mathrm{GL}}^{(\alpha)} s\left(x_{0}+1\right)$ has the same sign as $m$. That is, when $m>0$, we have $D_{\mathrm{GL}}^{(\alpha)} s\left(x_{0}\right)-D_{\mathrm{GL}}^{(\alpha)} s\left(x_{0}-1\right)>0$ and
$D_{\mathrm{GL}}^{(\alpha)} s\left(x_{0}\right)-D_{\mathrm{GL}}^{(\alpha)} s\left(x_{0}+1\right)>0$, and when $m<0$, we have $D_{\mathrm{GL}}^{(\alpha)} s\left(x_{0}\right)-D_{\mathrm{GL}}^{(\alpha)} s\left(x_{0}-1\right)<0$ and $D_{\mathrm{GL}}^{(\alpha)} s\left(x_{0}\right)-D_{\mathrm{GL}}^{(\alpha)} s\left(x_{0}+1\right)<0$. Thus, $x_{0}$ is the singularity.

Summarizing the above conclusion, we have the following.

Theorem 10. The fractional derivatives can detect and locate four types of ideal singularities correctly.

\section{Conclusions}

In this paper, we study fractional-order derivatives of lefthanded Grünwald-Letnikov formula with $0<\alpha<1$ to detect and locate singularities in theory. Theory analysis indicates that fractional-order derivatives of left-handed GrünwaldLetnikov formula with $0<\alpha<1$ can detect and locate the ideal four types of singularities correctly, which shows better performance than classical 1-order derivatives in theory.

\section{Conflict of Interests}

The authors declare that there is no conflict of interests regarding the publication of this paper.

\section{Acknowledgments}

This paper is supported by the National Natural Science Foundation of China (nos. 60873102 and 60873264), Major State Basic Research Development Program (no. 2010CB732501), and Open Foundation of Visual Computing and Virtual Reality Key Laboratory of Sichuan Province (no. J2010N03). This work was supported by a Grant from the National High Technology Research and Development Program of China (no. 2009AA12Z140).

\section{References}

[1] Y. Chen, Y. Li, W. Yu, L. Luo, W. Chen, and C. Toumoulin, "Jointmap tomographic reconstruction with patch similarity based mixture prior model," Multiscale Modeling and Simulation, vol. 9, no. 4, pp. 1399-1419, 2011.

[2] S. Hu, Z. Liao, D. Sun, and W. Chen, "A numerical method for preserving curve edges in nonlinear anisotropic smoothing," Mathematical Problems in Engineering, vol. 2011, Article ID 186507, 14 pages, 2011.

[3] Y. Chen, X. Yin, L. Shi et al., "Improving abdomen tumor low-dose CT images using a fast dictionary learning based processing," Physics in Medicine and Biology, vol. 58, no. 16, pp. 5803-5820, 2013.

[4] Z. Bian, J. Ma, J. Huang et al., "SR-NLM: a sinogram restoration induced non-local means image filtering for low-dose computed tomography," Computerized Medical Imaging and Graphics, vol. 37, no. 4, pp. 293-303, 2013.

[5] J. Ma, H. Zhang, Y. Gao et al., "Iterative image reconstruction for cerebral perfusion CT using a pre-contrast scan induced edgepreserving prior," Physics and Medical Biology, vol. 57, no. 22, pp. 7519-7542, 2012.

[6] Z. Jun and W. Zhihui, "A class of fractional-order multi-scale variational models and alternating projection algorithm for 
image denoising," Applied Mathematical Modelling, vol. 35, no. 5, pp. 2516-2528, 2011.

[7] J. Bai and X.-C. Feng, "Fractional-order anisotropic diffusion for image denoising," IEEE Transactions on Image Processing, vol. 16, no. 10, pp. 2492-2502, 2007.

[8] M. Janev, S. Pilipović, T. Atanacković, R. Obradović, and N. Ralević, "Fully fractional anisotropic diffusion for image denoising," Mathematical and Computer Modelling, vol. 54, no. 1-2, pp. 729-741, 2011.

[9] C. Wang, L. Lan, and S. Zhou, "Grünwald-Letnikov based adaptive fractional differential algorithm on image texture enhancing," Journal of Computational Information Systems, vol. 9, no. 2, pp. 445-454, 2013.

[10] H. A. Jalab and R. W. Ibrahim, "Texture enhancement based on the Savitzky-Golay fractional differential operator," Mathematical Problems in Engineering, vol. 2013, Article ID 149289, 8 pages, 2013.

[11] S. Hu, Z. Liao, and W. Chen, "Sinogram restoration for lowdosed X-ray computed tomography using fractional-order Perona-Malik diffusion," Mathematical Problems in Engineering, vol. 2012, Article ID 391050, 13 pages, 2012.

[12] Z. Liao, "Low-dosed x-ray computed tomography imaging by regularized fully spatial fractional-order Perona-Malik diffusion," Advances in Mathematical Physics, vol. 2013, Article ID 371868, 9 pages, 2013.

[13] Z. Liao, "A new definition of fractional derivatives based on truncated left-handedGrunwald-Letnikov formula with $0<$ $\alpha<1$ and median correction," Abstract and Applied Analysis. In press.

[14] M. Li, "A class of negatively fractal dimensional Gaussian random functions," Mathematical Problems in Engineering, vol. 2011, Article ID 291028, 18 pages, 2011.

[15] M. Li and W. Zhao, "On bandlimitedness and lag-limitedness of fractional Gaussian noise," Physica A: Statistical Mechanics and Its Applications, vol. 392, no. 9, pp. 1955-1961, 2013.

[16] M. Li, S. C. Lim, C. Cattani, and M. Scalia, "Characteristic roots of a class of fractional oscillators," Advances in High Energy Physics, vol. 2013, Article ID 853925, 7 pages, 2013.

[17] S. Hu, "External fractional-order gradient vector Perona-Malik diffusion for sinogram restoration of low-dosed x-ray computed tomography," Advances in Mathematical Physics, vol. 2013, Article ID 516919, 10 pages, 2013.

[18] D. A. Benson, S. W. Wheatcraft, and M. M. Meerschaert, "Application of a fractional advection-dispersion equation," Water Resources Research, vol. 36, no. 6, pp. 1403-1412, 2000.

[19] R. Hilfer, Applications of Fractional Calculus in Physics, vol. 463, World Scientific, Singapore, 2000.

[20] E. Sousa, "How to approximate the fractional derivative of order $1<\alpha \leq 2$," International Journal of Bifurcation and Chaos in Applied Sciences and Engineering, vol. 22, no. 4, Article ID 1250075, 13 pages, 2012.

[21] I. Podlubny, Fractional Differential Equations, Academic Press, San Diego, Calif, USA, 1999.

[22] S. G. Samko, A. A. Kilbas, and O. I. Marichev, Fractional Integrals and Derivatives: Theory and Applications, Gordon and Breach, London, UK, 1993.

[23] A. A. Kilbas, H. M. Srivastava, and J. J. Trujillo, Theory and Applications of Fractional Differential Equations, Elsevier, Amsterdam, The Netherlands, 2006. 


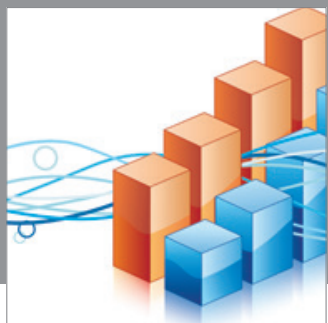

Advances in

Operations Research

mansans

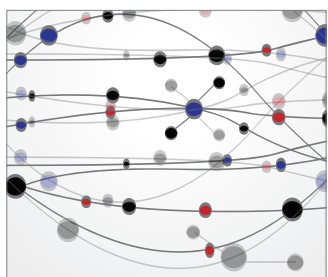

The Scientific World Journal
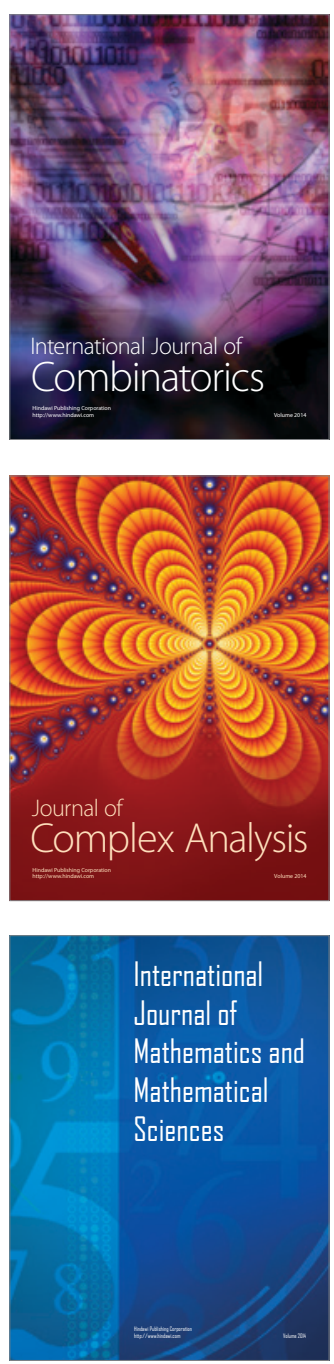
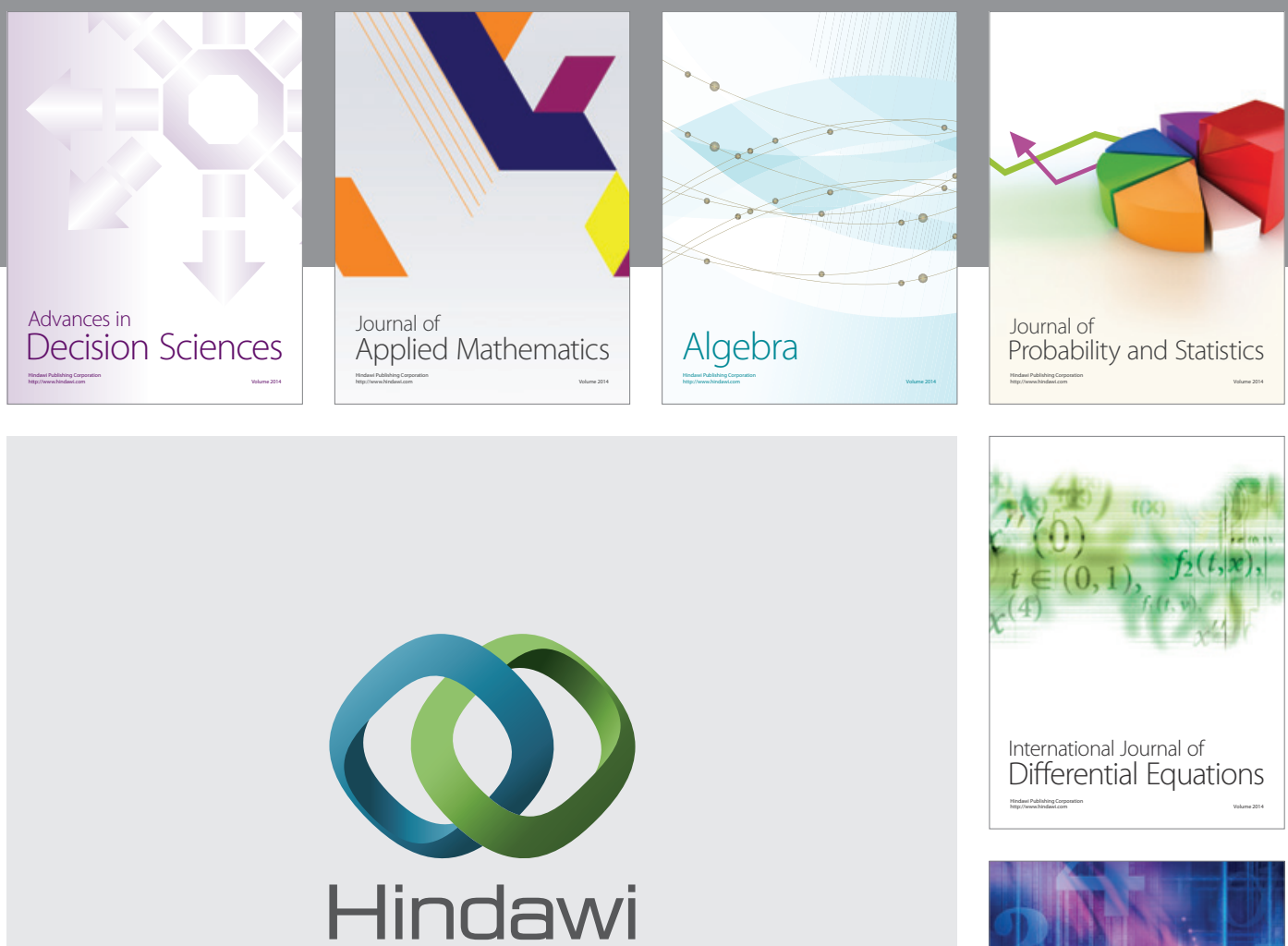

Submit your manuscripts at http://www.hindawi.com
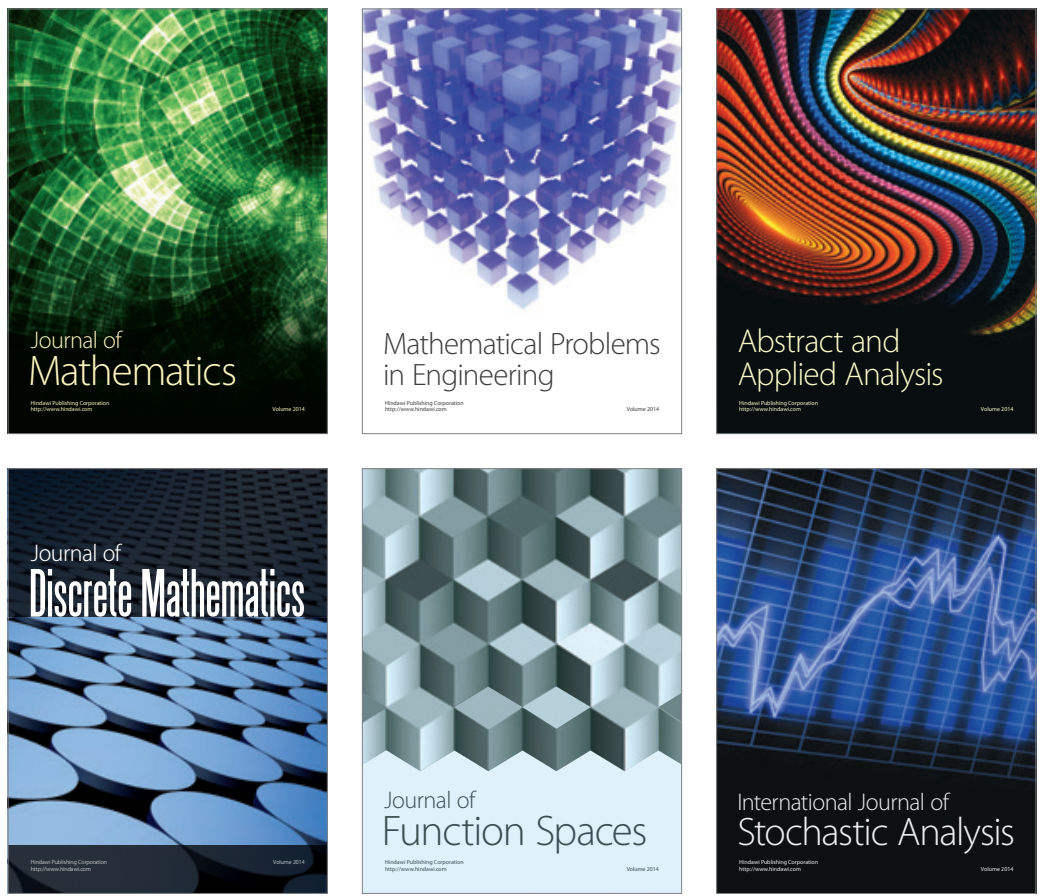

Journal of

Function Spaces

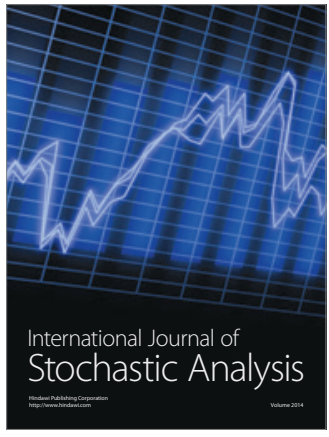

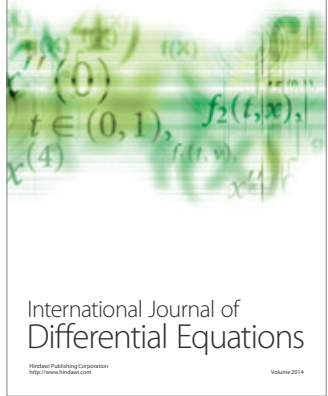
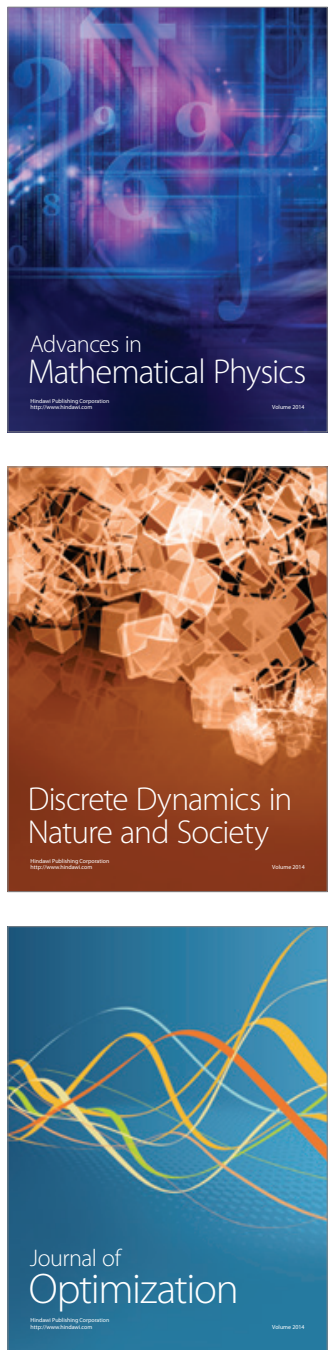